\title{
When weight loss is not self-motivated: Cognitive behavioral and medical nutrition therapy for weight management in a case of idiopathic intracranial hypertension
}

\author{
Emilia Vassilopoulou ${ }^{1 *}$, Dimitris Efthymiou ${ }^{2}$, Vardaka Elissavet ${ }^{1}$ and Agorastos Agorastos ${ }^{2}$ \\ ${ }^{1}$ Diet and Nutrition, International Hellenic University, Thessaloniki, Greece \\ ${ }^{2}$ Life and Health Sciences, Medical School, Aristotle University, Thessaloniki, Greece
}

\begin{abstract}
Background: Idiopathic Intracranial Hypertension (IIH) is a rare syndrome of elevated intracranial pressure occurring in obese adult women. Intracranial pressure occurring among the symptoms can lead to progressive irreversible visual loss and optic atrophy. Treatment demands weight loss and low-salt diet in order to preserve these health complications.

Case presentation: A case of a young adult woman with IIH and Class III obesity is described. Initially only personalized Medical Nutrition Therapy (MNT) was applied focused on the Mediterranean Diet. Due to low level of compliance Cognitive Behavioral Therapy (CBT) was introduced aiming to change beliefs on selfimage, self-capacity and eating and physical activity habits. Mediterranean Diet Score (MedDietScore), depression and anxiety were assessed throughout, together with standard anthropometric measurements. After one year the patient lost 10,5\% of initial weight, increased MedDietScore, scores of self-efficacy, self-esteem, whereas anxiety scores reduced.

Conclusions: Successful weight loss is of exceptional need for patients suffering from IIH. IIH mainly occurs in obese females of childbearing age, with significant cognitive distortions, built during childhood and puberty, regarding body image and weight. The case is a perfect example of the effective MNT and CBT intervention on obese patients, who unintentionally refer to a dietitian for weight loss.
\end{abstract}

Abbreviations: IIH: Idiopathic intracranial hypertension; MNT: Medical nutrition therapy; CBT: Cognitive behavioral therapy; MedDietScore: Meditteranean diet score; BDI II: Beck depression inventory II; BAI: Beck anxiety inventory.

\section{Introduction}

Idiopathic Intracranial Hypertension (IIH), also known as pseudotumor cerebri, is a rare syndrome of elevated intracranial pressure of unknown cause mainly occurring in obese females of childbearing age [1]. As it can be only diagnosed with exclusion of other disease, a detailed clinical history, imaging and cerebrospinal fluid examination are essential. Symptoms include intracranial pressure, including headaches and papilledema, which can result in progressive irreversible visual loss and optic atrophy. Treatment depends on the disease stage and aims to alleviate symptoms and preserve problems in vision. Additionally, weight management is recommended aiming a weight loss of at least 5-10\% in obese patients, along with a low-salt diet.

Herein we describe a young obese woman referred one year ago, with the diagnosis of IIT, in order to lose weight.

\section{Case presentation}

\section{Patient clinical history}

K.M is a 24 year old woman who visited the Emergency Care of the Hospital with intense, diffuse headache for the last two months and dull vision for the last 1.5 month, mainly focused on the right side. Informed consent was signed for evaluating and publishing data of intervention.

Neurological examination revealed no pathological data at entrance and exit of the hospital. The patient had no history of allergies, the Electrocardiogram shown sinus rhythm, and chest X-ray depicted lung areas in normal condition. Head MRI revealed optic nerve edema in both sides, slightly bigger in the right side, with no alterations in cerebral parenchyma, mid-line structures appeared undamaged, abdominal system and subarachnoid spaces of normal morphology and topography, as well as normal imaging of optic nerve.

Lumbar puncture showed increased pressure of cerebrospinal fluid ( $30 \mathrm{~cm} \mathrm{H} 2 \mathrm{O}$ column), with normal cell and biochemical measurements and negative culture for infections and virus. Brain MRV was also performed

${ }^{\star}$ Correspondence to: Emilia Vassilopoulou, Nutritional Sciences and Dietetics, International Hellenic University, Greece, E-mail: vassilopoulouemilia@gmail.com

Key words: obesity, idiopathic intracranial hypertension, dietary education, cognitive behavioral therapy

Received: March 13, 2019; Accepted: April 29, 2019; Published: May 03, 2019 
Vassilopoulou E (2019) When weight loss is not self-motivated: Cognitive behavioral and medical nutrition therapy for weight management in a case of idiopathic intracranial hypertension

The patient was started on acetazolamide $250 \mathrm{mg}$ twice a day, during hospitalization, resulting on reduction of symptomatology. After regular ophthalmic assessment the eye edema reduced. The patient left the hospital 6 days after entrance, with the recommendation to visit the hospital dietitian for further dietary and lifestyle changes.

\section{Dietary intervention}

At the first visit all important information regarding clinical, dietary and environmental data of the patient (Table 1) were collected in order to design the medical nutrition therapy (MNT) according to the Nutritional care process (NCPM) [2]. This included nutritional assessment, nutritional diagnosis, nutritional intervention and nutritional monitoring and evaluation. Signed informed consent was obtained by the patient at the beginning of the treatment. Compliance to Mediterranean Diet was assessed (MedDietScore) at the beginning and end of the treatment period [3]. The dietary therapeutic intervention aimed for an approximate of $15-20 \%$ weight loss (107- 99 $\mathrm{kg}$ respectively) over the next 1 year, based on appropriate nutritional education (Table 2).

\section{Cognitive behavioral therapy}

Two months after the initiation of the dietary intervention, Cognitive Behavior Therapy (CBT) was introduced in the therapeutic scheme in order to enhance the MNT process. CBT specifically focused on motivation for changing eating habits and physical activity routines. At the initial assessment the patient filled Beck Depression Inventory II (BDI -II) [4] and Beck Anxiety Inventory (BAI) [5] questionnaires scoring 15 and 27 respectively. CBT program was developed according to the Cooper/Fairburn approach to the treatment of obesity [6] organized in a series of treatment modules that included: 1. starting treatment: pre-admission phase; 2. establishing and maintaining weight loss; 3. encouraging acceptance, addressing realistic expectations to body weight, and addressing body image concerns; 4 . long-term weight maintenance; 5. Physical activity were implemented throughout the

Table 1. Nutritional Assessment using anthropometrics, clinical, demographic, environmental data

\begin{tabular}{|c|c|}
\hline Anthropometrics: & $\begin{array}{l}\text { Weight }=124 \mathrm{~kg} \\
\text { Height }=1.70 \mathrm{~m} \\
\mathrm{BMI}=42.9 \mathrm{~kg} / \mathrm{m} 2\end{array}$ \\
\hline Biochemical & NA \\
\hline Clinical & Diagnosis of IIH, headaches, dumb vision Acetazolamide $250 \mathrm{mg} / 2 *$ day \\
\hline Dietary & $\begin{array}{l}\text { From the Food Frequency Questionnaire regarding compliance with Meditteranean Diet: MedDietScore: } 8^{3} \\
\text { From both FFQ and } 24 \text {-hour recall: } \\
\text { Rare consumption of non refined cereals, legumes and red wine, low consumption of fruit and even lower of vegetables, increased consumption of cheese and red } \\
\text { meat, and use of olive oil on a daily base. } \\
\text { Increased consumption of white bread in a daily base, products from white flour ie pasta, cereals, } \\
\text { Increased consumption of salty products such as olives, whole fat cheese, cold cuts, ready made cheese pies, pizzas and croissants, potato chips, pop corn, packaged } \\
\text { fruit juices and soft drinks. Seed oils and mayonnaise part of her diet on a daily base. Eats visible fat from meat and usually cooking is in non-stich cookware. } \\
\text { Does not add salt in her food during meal, but often eats precooked meals or orders fast food. Takes a B12 supplement on a daily base for the last } 2 \text { years (without } \\
\text { a doctors/dietitians recommendation). } \\
\text { Daily intake (After analysis of } 24 \text {-hour recall: approximate daily intake } 3850 \mathrm{kcal}-43 \% \text { of fats }(28 \% \text { saturated), } 18 \% \text { protein, } 39 \% \text { carbohydrates }(18 \% \text { sugars }) \text { and } \\
\text { sodium intake: } 6000 \mathrm{mg} \text {. Water intake approximately } 850 \mathrm{ml} \text { per day. } \\
\text { Ideal BW }=45+(0.9 * 20)=63 \mathrm{~kg} \\
\text { Adapted BW }=(124+63) / 2=93.5=93.5 \\
\text { Energy Requirements: Harris Benedict }=66.47+(13.75 * \mathrm{wt} \text { in } \mathrm{kg})+(5.003 * 170 \mathrm{in} \mathrm{cm})-(6.755 * \text { age in yrs })=66.47+1.705+850.71+148.61=1920 \\
\text { When } 17 y e a r s \text { old tried alone to loose weight with no sufficient results }\end{array}$ \\
\hline Environmental & $\begin{array}{l}\text { Received primary education, single, unemployed, lives with her parents, does not exercise, walks only when goes for shopping, does the house cleaning on a daily } \\
\text { base, has limited social life. Obese mother with Type II Diabetes. Obese father with Hypertension. }\end{array}$ \\
\hline
\end{tabular}

Table 2. Structured nutritional intervention with specific goals aiming to increase patient's compliance

Aim

Substitute seed oils with olive oil

Avoid precooked meals

Increase consumption of fruit, vegetables fresh fruit, use, salads containing chicken and fish as main courses.

Increase beans consumption

Increase whole wheat products consumption

Reduce red meat consumption in twice monthly

Reduce sugar consumption with

Reduce salt consumption to $2500 \mathrm{mg}$ per day

Increase physical activity

\section{Indicative Suggestions}

Specific portion of olive oil to be added at the end of cooking

Ideas of easy to cook meals

Bigger portions to be cooked at once to be stored in the freezer

Prepared a list of acceptable fruits and vegetables and asked to be put in a visible place in the kitchen as a daily reminder

Apart from fresh fruits/vegetables to consume dried fruits without added sugar, baked vegetables, fruit and vegetable smoothies, or added in another snack or main course (eg yogurt,

Traditional recipes with beans

Veggie burgers with beans and vegetables (freezer for future use)

Bread from beans (chickpeas, lentils)

Salads containing beans

Substitute white with whole wheat bread, pasta, eat oats for breakfast, brown rice instead of white

Suggestions of fish, seafood and white meat tasty meals with fish and white meat products. Reduce consumption of red meat at only once every 2 weeks.

Prepare homemade sweets without sugar eg homemade marmalade made from dried fruits, cake with apple juice and raisins, homemade hazelnut jar with tahini and honey, baked apples with honey and cinnamon etc

Education on how to read food labels

Increase herbs while cooking for better taste

Moderate intensity walking for at least 20 minutes at start, aiming to increase to $45 \mathrm{~min}$ after 3months 7days per week 
program [7]. CBT was performed for 16 consecutive weeks. The psychological intervention focused on treating issues related to the significance of slow but stable rhythm of weight loss for the next 2 years, body image issues, enhance motivation for changes regarding everyday routines related to body weight and physical activity, prioritize changes set by the dietitian, enhance the significance of weight loss for treating symptoms of IIH, with a focus to the impaired vision and headaches.

\section{Outcomes}

KM was diagnosed with Class III obesity, due to inadequate nutritional education resulting to consumption of increased energy intake, big food portions and increased intake of food products high in fat, salt and simple dietary sugars as seen from the 24 hour recall and food frequency questionnaire. MedDietScore at start point was 18. The dietary therapeutic intervention aimed for an approximate of $15-20 \%$ weight loss (107- $99 \mathrm{~kg}$ respectively) over the next 1 year, based on appropriate nutritional education (Table 2). Sessions with the dietitian were repeated every 2 weeks for the first 6 months and once per month for the next 6 months. Nutritional intervention was designed to reduce portion sizes, fat, sugar and salt intake $(<2500 \mathrm{mg}$ sodium intake) and overall energy intake, increase consumption of dietary fiber, unsaturated fat and motivate patient to have a healthier lifestyle. Additionally, enhancement of cooking skills was aimed into a model adapted to the Mediterranean diet.

For the first three months the level of compliance was low, and the patient lost only $1 \mathrm{~kg}$ instead of the lowest aim for this period set of 5 $\mathrm{kg}$ loss. Obstacles derived from parental beliefs, previous unsuccessful effort to lose weight, as well as unmet expectations like faster weight loss and improvement on self-esteem.

The psychological intervention thereafter focused on treating issues related to the significance of slow but stable rhythm of weight loss for the next 2 years, body image issues, enhancement of motivation for changes regarding everyday routines related to body weight and physical activity, prioritization of changes set by the dietitian, enhancement of the significance of weight loss for treating symptoms of IIH, with a focus to the impaired vision and headaches.

Throughout the consultation appropriate behaviors were rewarded, negative beliefs that could generate negative conclusions, anxiety, depression and guilt were explored. The patient was motivated to change her beliefs about general self-efficacy and those related to weight change and control. After the end of the CBT sessions she filled again BDI-II and BAI questionnaires scoring 12 at BDI-II and only 8 at BAI, showing significant improvement on her anxiety level. Afterwards, some motivation interview strategies were included in the medical nutrition therapy [8]. After the fourth month of the initial visit and from the first month of CBT, the patient became more cooperative with the dietitian, and accepted suggestions regarding dietary and activity routines.

At the end of the first year from the initial visit the patient lost $26 \%$ of the initial weight $(96 \mathrm{~kg}$ ) and increased her MedDietScore to 38 . Additionally, initial symptoms of dull vision and headaches, principal symptoms of IIH, did not reoccur during the period of intervention.

\section{Discussion and conclusion}

A case of a young obese woman diagnosed with IIT and the underlying effectiveness of a structured medical nutrition therapy when combined with CBT for obesity is described herein.
CBT is well recognized as effective for the psychological intervention in eating disorders sufferers. It has been previously discussed for treating obese patients in order to loose weight, avoid invasive medical procedures and also minimize subsequent weight regain [7].

Motivational interview has also been previously discussed for adding effectiveness to the weight loss trip [9], but still research is needed in the area, as previous mode of application appears with methodological insufficiencies [10]. Nevertheless, in our case we found that CBT was of favor for the initial intervention, as the patient suffered from significant distortions from past experiences and beliefs for own-self regarding among others body image and diet management. Motivational interviewing as a secondary intervention can add value to both the MNT and CBT.

Medical nutrition therapy of obese people is important to educate patients into healthier dietary models and lifestyle behaviors. This way weight loss is promoted and weight regain obstructed. Cognitive behavioral therapy should be in parallel part of the intervention program, in order to enhance self esteem and capability of the patient to recognize previous experiences or habits, related to dietary habits and body image, that may inhibit or cause problems into sustaining weight loss [11]. Cognitive behavioral therapy (CBT) is traditionally recognized as the best established treatment for binge eating disorder and the most preferred intervention for obesity, and could be considered as the firstline treatment among psychological approaches, especially in a longterm perspective; however, it does not necessarily produce a successful weight loss [12]. For the long term and follow up period, motivational interviewing can add effectiveness to weight loss $[8,13,14]$.

To sum up with, herein we describe a case of a young adult woman suffering from IIH, due to obesity stage III, who benefit from the combination of MNT and CBT. Limited knowledge of healthy eating, previous unsuccessful weight loss experiences and low selfesteem build tough barriers for successful weight loss. Weight and stress related to weight management, body image and self-esteem were significantly reduced. Severe anxiety level reduced after the CBT intervention, resulting on significant effort of the patient to change eating and physical activity habits. This is a typical example of patients referring to a dietitian, after a doctor's recommendation for weight loss, due to the serious health complications of increased body weight. As these patients need additional motivation and education to adapt into a new healthier model of life, intense MNT and CBT appear more than promising into dissolving distortions and achieving long term dietary and physical activity changes.

\section{Declarations}

Ethics approval and consent to participate: Hospital's ethics committee approved the study and informed consent from the patient was received.

Consent for publication: Consent for publication was received by the patient.

Availability of data and materials: The datasets used and/or analysed during the current study are available from the corresponding author on reasonable request.

Competing interests: The authors declare that they have no competing interests

Funding: The authors received no specific funding for this work. 


\section{Acknowledgments: Not applicable}

Author contributions: Emilia Vassilopoulou is an Assistant Professor of Diet and Nutrition at International Hellenic University. She was responsible for all nutrition interventions performed at the described case.

Dimitris Efthymiou is a Medical Doctor, practising his specialty as a Psychiatrist and was responsible for the CBT intervention to the case.

Elissavet Vardaka is an Associate Professor of Diet and Nutrition at International Hellenic University. She participated in the nutritional monitoring of the patient.

Agorastos Agorastos is a Psychiatrist, Assistant Professor of Psychiatry at Aristotle University of Thessaloniki who supervised the CBT's interventions.

\section{References}

1. Thurtell MJ, Wall M (2013) Idiopathic intracranial hypertension (pseudotumor cerebri): recognition, treatment, and ongoing management. Curr Treat Options Neurol 15: 1-12. [Crossref]

2. Writing group of the nutrition care process/standardized language committee (2008) Nutrition care process and model part I: The 2008 update. J Am Diet Assoc 108: 11131117. [Crossref]

3. Kontou N, Psaltopoulou T, Soupos N, Polychronopoulos E, Xinopoulos D, et al. (2013) The mediating effect of Mediterranean diet on the relation between smoking and colorectal cancer: a case-control study. Eur J Public Health 23: 742-746. [Crossref]

4. Beck AT, Steer RA, Carbin MG (1988) Psychometric properties of the Beck Depression Inventory: Twenty-five years of evaluation. Clin Psychol Rev 8: 77-100.
5. Beck AT, Epstein N, Brown G, Steer RA (1988) An inventory for measuring clinical anxiety: Psychometric properties. J Consult Clin Psychol 56: 893-897. [Crossref]

6. Cooper Z, Fairburn C, Hawker D (2003) Cognitive-behavioral treatment of obesity: A clinician's guide.

7. Fennig S, Fennig S (2006) Can we treat morbid obese children in a behavioral inpatient program? Pediatr Endocrinol Rev 3: 590-596. [Crossref]

8. Barrett S, Begg S, O'Halloran P, Kingsley M (2018) Integrated motivational interviewing and cognitive behaviour therapy for lifestyle mediators of overweight and obesity in community-dwelling adults: a systematic review and meta-analyses. $B M C$ Public Health 18: 1160. [Crossref]

9. Flattum C, Friend S, Neumark-Sztainer D, Story M (2009) Motivational interviewing as a component of a school-based obesity prevention program for adolescent girls. $J \mathrm{Am}$ Diet Assoc 109: 91-94. [Crossref]

10. Smith DE, Heckemeyer CM, Kratt PP, Mason DA (1997) Motivational interviewing to improve adherence to a behavioral weight-control program for older obese women with NIDDM. A pilot study. Diabetes Care 20: 52-54. [Crossref]

11. Copperman N, Jacobson MS (2003) Medical nutrition therapy of overweight adolescents. Adolesc Med 14: 11-21. [Crossref]

12. Castelnuovo G, Pietrabissa G, Manzoni GM, Cattivelli R, Rossi A, et al. (2017) Cognitive behavioral therapy to aid weight loss in obese patients: Current perspectives. Psychol Res Behav Manag 10: 165-173. [Crossref]

13. Barrett S, Begg S, O'Halloran P, Kingsley M (2018) Integrated motivational interviewing and cognitive behaviour therapy can increase physical activity and improve health of adult ambulatory care patients in a regional hospital: the Healthy $4 \mathrm{U}$ randomised controlled trial. BMC Public Health 18: 1166. [Crossref]

14. Patnode CD, Evans CV, Senger CA, Redmond N, Lin JS (2017) Behavioral counseling to promote a healthful diet and physical activity for cardiovascular disease prevention in adults without known cardiovascular disease risk factors. JAMA 318: 175. [Crossref]

Copyright: (C2019 Vassilopoulou E. This is an open-access article distributed under the terms of the Creative Commons Attribution License, which permits unrestricted use, distribution, and reproduction in any medium, provided the original author and source are credited. 